\title{
An Exact Solution for Quantum Tunneling in a Dissipative System *
}

\author{
Li Hua Yu \\ National Synchrotron Light Source, Brookhaven National Laboratory, N.Y.11973
}

\begin{abstract}
Applying a technique developed recently $[1,2]$ for an harmonic oscillator coupled to a bath of harmonic oscillators, we present an exact solution for the tunneling problem in an Ohmic dissipative system with inverted harmonic potential. The result shows that while the dissipation tends to suppress the tunneling, the Brownian motion tends to enhance the tunneling. Whether the tunneling rate increases or not would then depend on the initial conditions. We give a specific formula to calculate the tunneling probability determined by various parameters and the initial conditions.
\end{abstract}

\section{DISCLAIMER}

\begin{abstract}
This report was prepared as an account of work sponsored by an agency of the United States Government. Neither the United States Government nor any agency thereof, nor any of their employees, makes any warranty, express or implied, or assumes any legal liability or responsibility for the accuracy, completeness, or usefulness of any information, apparatus, product, or process disclosed, or represents that its use would not infringe privately owned rights. Reference herein to any specific commercial product, process, or service by trade name, trademark, manufacturer, or otherwise does not necessarily constitute or imply its endorsement, recommendation, or favoring by the United States Government or any agency thereof. The views and opinions of authors expressed herein do not necessarily state or reflect those of the United States Government or any agency thereof.
\end{abstract}

*Work Performed Under The Auspices of the U.S. Dept. of Energy 


\section{Introduction.}

Quantum tunneling with dissipation has been studied by many people after the work by Caldeira and Leggett[3-6]. These studies use different approximations to calculate the tunneling probability. Among these works, there is a widely discussed question about whether the dissipation suppresses or enhances the quantum tunneling[7]. In this paper, we shall show that for a special potential barrier, the inverted harmonic potential well, the tunneling problem can be solved exactly, thus we shall answer this question precisely. The technique we used for this solution has been developed recently to solve the wavefunction evolution for another dissipative system problem: an harmonic oscillator coupled to an environment $[1,2]$. In this paper we shall show that this technique is equally applied to the inverted harmonic potential.

The simplest example of a dissipative system, an harmonic oscillator coupled to an environment of a bath of harmonic oscillators, has been the subject of extensive studies (see [1] for references). In a recent paper[1], we obtained a simple and exact solution for the wave function of the system plus the bath, in the Ohmic case. It is described by the direct product in two independent Hilbert spaces. One of them is described by the Caldirola-Kanai (CK) Hamiltonian, the other represents the effect of the bath, i.e., the Brownian motion. In a second paper[2], using this wavefunction solution, we derived a simple formula for the probability distribution at finite temperature, expressed in terms of the wavefunction solution for the CK Hamiltonian only.

In this paper we shall apply these results to a different system, the inverted harmonic potential well to study the quantum tunneling problem of dissipative system. We shall use

the same notations as the reference $[1,2]$ and quote formulas from there. The Hamiltonian of this system is: 


$$
H=\frac{p^{2}}{2 M}-\frac{1}{2} M\left(\omega_{0}^{2}+\Delta \omega^{2}\right) q^{2}+q \sum_{j} c_{j} x_{j}+\sum_{j}\left(\frac{p_{j}^{2}}{2 m_{j}}+\frac{1}{2} m_{j} \omega_{j}^{2} x_{j}^{2}\right) .
$$

The only difference of this Hamiltonian from that in reference [1] is the sign of the second term of the right hand side, i.e., the potential is now an inverted harmonic potential well. Assuming the Ohmic bath oscillator density to be the same as given in [1]:

$$
\rho\left(\omega_{j}\right)=\frac{2 \eta \dot{M}}{\pi} \frac{m_{j} \omega_{j}^{2}}{c_{j}^{2}}
$$

Following exactly the same procedure as [1], we derive the Langevin equation of motion for the system:

$$
\ddot{q}+\eta \dot{q}-\omega_{0}^{2} q=f(t),
$$

with the Brownian motion driving force:

$$
f(t)=-\sum_{j} \frac{c_{j}}{M}\left(x_{j 0} \cos \omega_{j} t+\dot{x}_{j 0} \frac{\sin \omega_{j} t}{\omega_{j}}\right) .
$$

This equation is easily solved to give:

$$
q(t)=a_{1}(t) q_{0}+a_{2}(t) \dot{q}_{0}+\sum_{j}\left(b_{j 1}(t) x_{j 0}+b_{j 2}(t) \dot{x}_{j 0}\right),
$$

where $q_{0}, \dot{q}_{0}, x_{j 0}, \dot{x}_{j 0}$ are the initial position and velocity operators of the system and bath respectively, and

$$
\begin{gathered}
a_{1}=e^{-\frac{\eta}{2} t}\left(\cosh \omega t+\frac{\eta}{2 \omega} \sinh \omega t\right), a_{2}=e^{-\frac{\eta}{2} t} \frac{\sinh \omega t}{\omega} \\
b_{j 1}(t)=-\frac{c_{j}}{M} \int_{0}^{t} a_{2}\left(t^{\prime}\right) \cos \omega_{j}\left(t-t^{\prime}\right) d t^{\prime}, b_{j 1}(t)=-\frac{c_{j}}{M} \int_{0}^{t} a_{2}\left(t^{\prime}\right) \sin \omega_{j}\left(t-t^{\prime}\right) d t^{\prime},
\end{gathered}
$$

with $\omega=\left(\omega_{0}^{2}+\frac{\eta^{2}}{4}\right)^{1 / 2}$.

Then, based on the same arguments of [1], this solution can be used to show that the wavefunction of the system plus the bath can be written as

$$
\Psi\left(q,\left\{\xi_{j}\right\}, t\right)=\psi\left(q-\sum_{j} \xi_{j}, t\right) \prod_{j=1}^{N} \chi_{j}\left(\xi_{j}, t\right)
$$


where the wavefunction $\psi(Q, t)$ is a solution of Schoedinger equation with the time dependent CK Hamiltonian

$$
H_{Q}=e^{-\eta t} \frac{P^{2}}{2 M}-\frac{1}{2} M \omega_{0}^{2} e^{\eta t} Q^{2}
$$

and the commutation $[Q, P]=i \hbar$, while $\xi_{j}=b_{j 1}(t) x_{j 0}+b_{j 2}(t) \dot{x}_{j 0}$ is the contribution of the $\mathrm{j}$ 'th bath oscillator to the Brownian motion. The function $\chi_{j}\left(\xi_{j}, t\right)$ is given by $\chi_{j}\left(\xi_{j}, t\right)=$ $<\theta_{\xi_{j}}\left|\chi_{j 0}\right\rangle$, where $\chi_{j 0}\left(x_{j 0}\right)$ is the initial state of the bath oscillator, and $\mid \theta_{\xi_{j}}>$ is an eigenstate of the operator $\xi_{j}$ with eigenvalue $\xi_{j}$, as given by eq.(4) of [2]:

$$
\theta_{\xi_{j}}\left(x_{j 0}, t\right)=\left(\frac{m_{j}}{2 \pi \hbar b_{j 2}}\right)^{\frac{1}{2}} \exp \left[-\frac{i m_{j}}{2 \hbar b_{j 2}}\left(b_{j 1} x_{j 0}^{2}-2 x_{j 0} \xi_{j}\right)\right] .
$$

According the analysis of [2], assuming the bath is at temperature $T$, averaging over the Boltzmann distribution of the wavefunction $\chi_{j 0}\left(x_{j 0}\right)$ of the bath oscillators, and using eq.(8), we find the probability distribution the same as derived in [2]:

$$
\rho(q, t)=\frac{1}{\sqrt{2 \pi} \sigma_{\xi}} \int|\psi(q-\xi, t)|^{2} e^{-\frac{\xi^{2}}{2 \sigma_{\xi}^{2}}} d \xi
$$

where $\sigma_{\xi}$ is the Brownian motion width:

$$
\sigma_{\xi}^{2}(t)=\int_{0}^{\omega_{c}} \frac{\hbar}{2 m_{j} \omega_{j}}\left(\left|b_{j 1}(t)\right|^{2}+\omega_{j}^{2}\left|b_{j 2}(t)\right|^{2}\right) \operatorname{coth}\left(\frac{\hbar \omega_{j}}{2 k T}\right) \rho\left(\omega_{j}\right) d \omega_{j} .
$$

This result is apparently the same as that given in [2], but the expressions for $b_{j 1}(t)$ and $b_{j 2}(t)$ are different. There are also subtleties associated with a logarithmic divergence of the integration over $\omega_{j}$, which is removed by introducing a cut-off frequency $\omega_{c}$. This width is zero initially, but then increases exponentially to infinity as time evolves. In the following, for simplicity, we consider only the low temperature limit, then the width approaches in a time range of about $1 / \omega$ to the asymptotic value

$$
\sigma_{\xi}^{2}=e^{(2 \omega-\eta) t} \sigma_{0}^{2} \frac{\eta}{2 \omega} \frac{\omega_{0}}{\omega} B
$$


with $\sigma_{0}^{2} \equiv \hbar /\left(2 M \omega_{0}\right)$ (the width of the ground state of an harmonic oscillator with frequency $\left.\omega_{0}\right)$ and

$$
B \equiv \frac{1}{\pi} \ln \left[1+\left(\frac{\omega_{c}}{\omega-\frac{\eta}{2}}\right)^{2}\right]
$$

2. The wavefunction evolution

To calculate the tunneling probability, we assume that initially the wavefunction is a gaussian wave packet centered at the right of the peak of the potential by $z_{0}$ with a velocity $v_{0}=-\hbar k / M$ towards the potential barrier peak:

$$
\psi_{0}(q) \equiv \psi(q ; t=0)=\left(2 \pi \sigma^{2}\right)^{-1 / 4} e^{-\frac{\left(q-z_{0}\right)^{2}}{4 \sigma^{2}}+i k q}
$$

First, following exactly the same method in [1], we derive the Green's function of the time dependent Hamiltonian eq.(9):

$$
G\left(q, q_{0} ; t, 0\right)=\left(\frac{M}{2 \pi i \hbar a_{2}}\right)^{\frac{1}{2}} \exp \left[\frac{i M}{2 \hbar a_{2}}\left(a_{1} q_{0}^{2}+\dot{a}_{2} e_{1}^{\eta t} q^{2}-2 q_{0} q\right)\right] .
$$

Then we calculate the wavefunction $\psi(q, t)$ :

$$
\begin{aligned}
\psi(q, t) & =\int G\left(q, q_{0} ; t, 0\right) \psi_{0}\left(q_{0}\right) d q_{0} \\
& =\left(2 \pi \sigma^{2}\right)^{-1 / 4}\left(a_{1}+i \omega_{0} a_{2} r^{2}\right) e^{-\frac{\left(q-z_{0}\right)^{2}}{4 \sigma^{2}}+i\left(c_{2} q^{2}+c_{1} q+c_{0}\right)}
\end{aligned}
$$

This result represents a gaussian distribution centered at $q_{c}=a_{1} z_{0}-a_{2} v_{0}$ (the classical trajectory of a particle initially at $z_{0}$ with velocity $-v_{0}$ ) and with a width of

$$
\sigma_{\theta}^{2} \equiv \sigma^{2}\left(a_{1}^{2}+r^{4} \omega_{0}^{2} a_{2}^{2}\right)
$$

where $r \equiv \sigma_{0} / \sigma$. The coefficients in the phase factor exponent are: 


$$
\begin{gathered}
c_{2}=\frac{M e^{\eta t}}{4 \hbar} \frac{d}{d t}\left(\ln \sigma_{\theta}^{2}\right), \\
c_{1}=\frac{M e^{\eta t}}{4 \hbar}\left(-2 q_{c} \frac{d}{d t}\left(\ln \sigma_{\theta}^{2}\right)+4 \dot{q}_{c}\right), \\
c_{0}=\frac{k a_{2}}{4} e^{\eta t}\left(q_{c} \frac{d}{d t}\left(\ln \sigma_{\theta}^{2}\right)-2 \dot{q}_{c}\right)+\frac{q_{c} z_{0}}{4 \sigma_{\theta}^{2}} \omega_{0} a_{2} r^{2} .
\end{gathered}
$$

This phase factor is related to the current density, as will be explained later. It is straight forward to verify that eq.(18) indeed satisfies the Schoedinger equation with the time dependent Hamiltonian eq.(9). With these provisions, we are ready to calculate the tunneling probability.

3. The tunneling probability and current density

Using the density eq.(11) and wavefunction eq.(18), we find a very simple expression for the probability density:

$$
\rho(q, t)=\frac{1}{\sqrt{2 \pi} \sigma_{t}} e^{-\frac{(q-q c)^{2}}{2 \sigma_{t}^{2}}},
$$

where

$$
\sigma_{t}^{2} \equiv \sigma_{\theta}^{2}+\sigma_{\xi}^{2}
$$

is the total width including the Brownian motion width.

The probability for the particle to pass to the left of position $q$ at $t$ is then:

$$
P(q, t)=\int_{-\infty}^{q} \rho\left(q^{\prime}, t\right) d t^{\prime}=F\left(\frac{q-q_{c}}{\sqrt{2} \sigma_{t}}\right)
$$

where the function $F$ is:

$$
F(W) \equiv \frac{1}{\sqrt{\pi}} \int_{-\infty}^{W} e^{-u^{2}} d u
$$


Hence the current density is found to be:

$$
I=-\frac{\partial P(q, t)}{\partial t}=|\psi|^{2}\left(\frac{1}{2}\left(q-q_{c}\right) \frac{d}{d t}\left(\ln \sigma_{t}^{2}\right)+\dot{q}_{c}\right) .
$$

On the other hand, if the Brownian motion can be ignored (replacing $\sigma_{t}$ by $\sigma_{\theta}$ ), the solution eq.(18) of the time dependent Schoedinger equation should also satisfy the expression for the current density:

$$
I=\frac{\hbar}{2 M e^{\eta t}}\left(\psi \frac{\partial \psi^{*}}{\partial q}-\psi^{*} \frac{\partial \psi}{\partial q}\right)=\frac{\hbar}{M e^{\eta t}}|\psi|^{2}\left(2 c_{2} q+c_{1}\right) .
$$

The expressions for $c_{1}$ and $c_{2}$ eq.(21) and eq.(20) indeed satisfy eq.(27), hence they acquire the following clear physical meaning. If we denote the velocity of the "fluid" distribution $|\psi|^{2}$ as $v$, the current density is then $I=|\psi|^{2} v$. Therefore a comparison of eq.(27) with eq.(28) leads to:

$$
v=\frac{\hbar}{M e^{\eta t}}\left(2 c_{2} q+c_{1}\right)=\frac{1}{2}\left(q-q_{c}\right) \frac{d}{d t}\left(\ln \sigma_{\theta}^{2}\right)+\dot{q}_{c} .
$$

Thus those particles with larger $q$ acquire larger velocity falling from the apex of the potential. Since the width $\sigma_{\theta}^{2}$ is proportional to $\exp (2 \omega-\eta) t$, as time evolves, most particles move rapidly away from the apex of the potential. In the mean time, for any fixed $q \neq 0$, the wavenumber $2 c_{2} q+c_{1}$ also increases exponentially by a factor $e^{\eta t}$ according to eq.(21) and eq.(20) because the equivalent mass is $M e^{\eta t}$ instead of $M$. Hence the wavelength decays exponentially, as pointed out by reference [2]. When $t \gg 1 / \eta$ the system approaches the classical limit.

\section{Tunneling probability as time approaches infinity}

We define the tunneling probability $P$ as the probability for the particle to be at the left of the peak. Then, applying eq.(25), eq.(24) and eq.(19), we find $P=F(-W)$, with

$$
W=\frac{q_{c}}{\sqrt{2} \sigma_{t}}=\frac{a_{1} z_{0}-a_{2} v_{0}}{\sqrt{2\left(\sigma^{2}\left(a_{1}^{2}+r^{4} \omega_{0}^{2} a_{2}^{2}\right)+\sigma_{\xi}^{2}\right)}} .
$$


Because $a_{1}, a_{2}$, and $\sigma_{\xi}$ are all proportional to $e^{(\omega-\eta / 2) t}$ as time approaches infinity, $W$ approaches a finite limit, which determines the final tunneling probability. To simplify the expression for this limit, we use the following scaled variables:

$$
Z \equiv \frac{z_{0}}{\sigma} ; V \equiv \frac{v_{0}}{\omega_{0} z_{0}} ; \epsilon \equiv \frac{\eta}{2 \omega} ; r \equiv \frac{\sigma_{0}}{\sigma} ; B \equiv \frac{1}{\pi} \ln \left[1+\left(\frac{\omega_{c}}{\omega-\frac{\eta}{2}}\right)^{2}\right]
$$

Then, using the eq.(6) for $a_{1}, a_{2}$, and eq.(13) for the Brownian motion width, we have:

$$
W=\frac{Z}{\sqrt{2}} \frac{1-V \sqrt{\frac{1-\epsilon}{1+\epsilon}}}{\sqrt{1+\frac{1-\epsilon}{1+\epsilon} r^{4}+4 B \epsilon \sqrt{1-\epsilon^{2}} r^{2}}} .
$$

To understand the meaning of this expression, we remark that $Z \gg 1$ represents a case where the wavepacket width is much smaller than its distance from the origin. We also remark that if $\eta=0$, as time approaches infinity, $q_{c}=e^{\omega_{0} t}\left(z_{0}-v_{0} / \omega_{0}\right) / 2$. Hence $V<1$ means that a classical particle with initial velocity $v_{0}$ and position $z_{0}$ does not have enough kinetic energy to pass the potential barrier if there is no dissipation. Thus $Z \gg 1$ and $V<1$ represent a case relevant to the quantum tunneling problem.

The term $4 B \epsilon \sqrt{1-\epsilon^{2}} r^{2}$ comes from the Brownian motion. If $\epsilon \ll 1$, i.e., if the dissipation is small, this term increases as $\epsilon$ increases, and reduces $W$, which in turn increases $F(-W)$, hence enhances the tunneling. This effect is reduced if $r \ll 1$, or, in other words, if the initial wavepacket width is much larger than $\sigma_{0}$, the increase due to The Brownian motion is insignificant. If the initial velocity is not zero, the second term in the numerator increase $W$ as $\epsilon$ increases, thus the damping suppresses the tunneling. Intuitively this can be explained as that the damping makes the classical particle unable to move to the barrier peak as close as if there is no damping, and hence increases the barrier height.

To get an idea about the effect of dissipation on the tunneling probability, in figure 1 , we plot $P$ as a function of $V$ and $\epsilon$ for $Z=3, r=0.3$, and $B=3$, which corresponds to a cut-off frequency about 100 time larger than $\omega$. Because the logarithmic dependence of $B$ on $\omega_{c}$, the 
result is very insensitive to the cut-off frequency. The plot clearly shows that when $V$ is large, the tunneling is suppressed by the dissipation, while if initial velocity is zero, the dissipation enhances the tunneling.

\section{Tunneling probability and uncertainty principle}

Finally, it is interesting to examine the relation between the initial momentum and position distribution and the tunneling probability. For simplicity, we consider here only the case without dissipation. The Fourier transform of initial wavefunction eq.(15), the wavefunction in momentum space is:

$$
\psi_{0}(k)=\left(\frac{2 \sigma^{2}}{\pi}\right)^{1 / 4} e^{-\left(k+k_{0}\right)^{2}-i\left(k+k_{0}\right) z_{0}} .
$$

Thus from the classical point of view, the probability for the particle to pass the potential barrier is the probability of the initial velocity $v<-\omega_{0} z_{0}$, or $k<-z_{0} / 2 \sigma_{0}^{2}$ (notice that $v=\hbar k / M)$ :

$$
P=\int_{-\infty}^{-\frac{z_{0}}{2 \sigma_{0}^{2}}}\left|\psi_{0}(k)\right|^{2} d k=F\left(-\frac{Z(1-V)}{\sqrt{2} r^{2}}\right)
$$

A comparison with eq.(32) shows that if $r \gg 1$, i.e., if the initial width $\sigma$ is much smaller than the minimum wavepacket width $\sigma_{0}$, or, in other words, if the initial momentum spread is very large, this crude estimate is correct.

Another extreme is when $r \ll 1$, i.e., when we can consider the initial momentum spread is very small, and all the particles have velocity $v_{0}$, but initial position has large spread. Then from the classical point of view, the probability for particle to pass the potential barrier is its initial $q<v_{0} / \omega_{0}$, i.e.:

$$
P=\int_{-\infty}^{v_{0} / \omega_{0}}\left|\psi_{0}(q)\right|^{2} d q=F\left(-\frac{Z(1-V)}{\sqrt{2}}\right)
$$


Again, a comparison with eq.(32) shows that if $r \ll 1$, i.e., if the initial width $\sigma$ is much larger than the minimum wavepacket width $\sigma_{0}$, this crude estimate is also correct. These estimates indicate that the tunneling probability has a very simple relation with the uncertainty principle, and we have a very simple way to estimate the tunneling probability.

\section{Acknowledgments}

The author thanks Prof. C.N. Yang for many sessions of stimulating discussions. The author also likes to thank C.P. Sun for interesting discussions. The work is performed under the auspices of the U.S. Department of Energy under Contract No. DE-AC02-76CH00016. 


\section{References}

1. L.H.Yu, C.P.Sun, Phys. Rev. A 49, 592 (1994)

2. L.H.Yu, Phys. Rev.Lett. A 202, 167, (1995)

3. A.O. Caldeira, A.J. Leggett, Phys. Rev. Lett. 46, 211 (1981); Ann. Phys. 149, 374 (1983)

4. A.J.Bray and M.A.Moore, Phys. Rev. Lett. 46, 1545 (1982); S. Chakravarty, ibid. 49, 681 (1982);

5. G. Schön and A.D.Zaikin, phy. Rep. 198, 237 (1990), and references therein.

6. S. Chakravarty and A.J. Legget, Phys. Rev. Lett. 52, 5 (1984); A.J.Leggett, S. Chakravarty, A.T. Dorsey, M.P.A.Fisher, A.Garg, and W. Zweger, Rev. Mod.Phys. $59,1(1987)$.

7. About the discussion on whether the dissipation suppresses or enhance the quantum tunneling, see for example: A. J. Leggett; Satoshi Iso; in "Proceedings of the 4th International Symposium: Foundation of Quantum Mechanics in the Light of New Technology"; K. Fujikawa, S. Iso, M. Sasaki, and H. Suzuki, Phys. Rev. Lett. 68, 1093 (1992). 


\section{Figure Captions}

Figure 1. The tunneling probability as a function of the scaled dissipation coefficient $\epsilon$ and the scaled initial velocity $V$, assuming the scaled initial position $Z=3$, the scaled inverse wavepacket size $r=0.3$, and the scaled cut-off frequency $B=3$. 


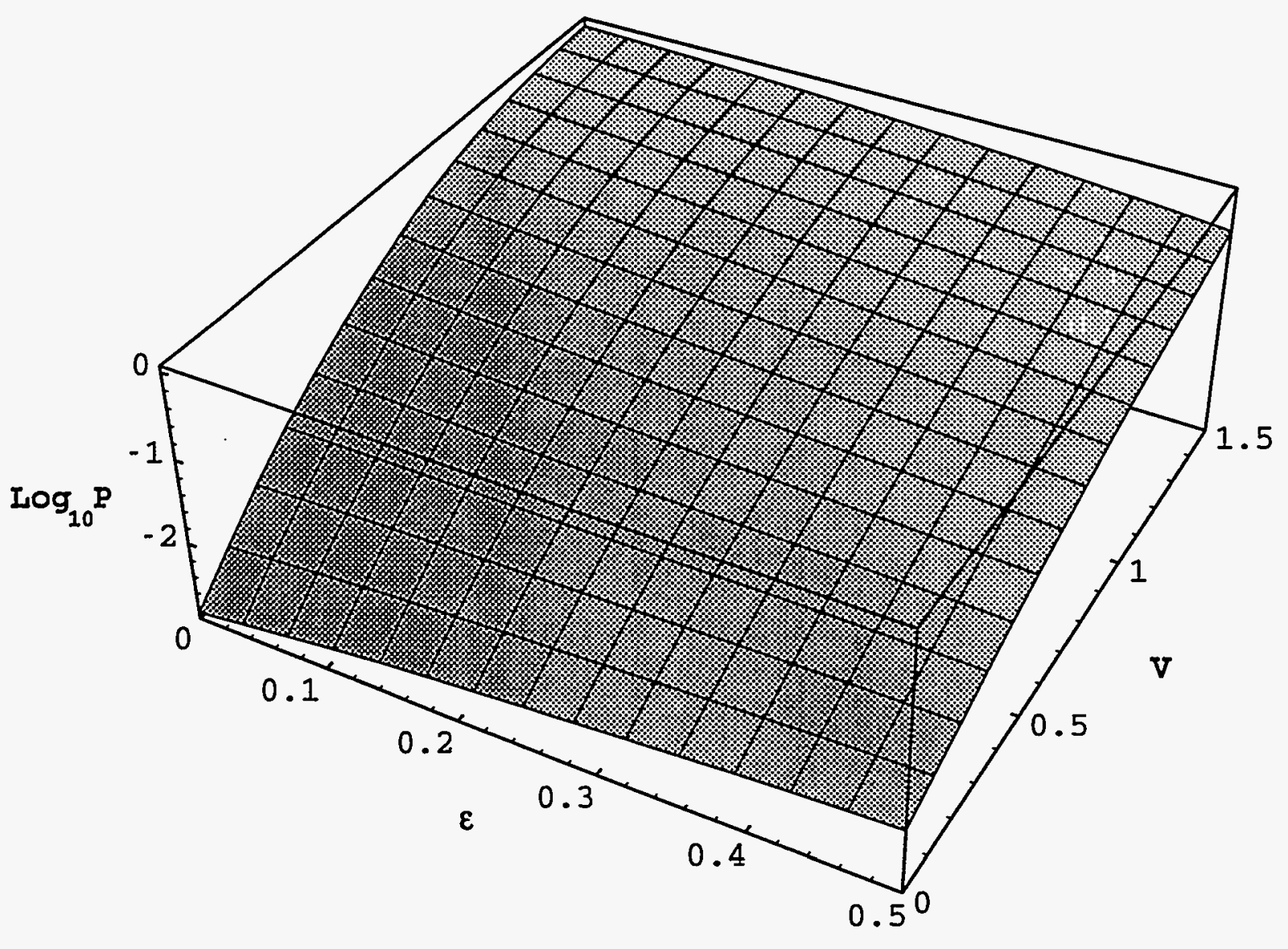

